\title{
Chapter 3 \\ Quantum Optics: Colloidal Fluorescent Semiconductor Nanocrystals (Quantum Dots) in Single-Molecule Detection and Imaging
}

\author{
Laurent A. Bentolila, Xavier Michalet, and Shimon Weiss(四)
}

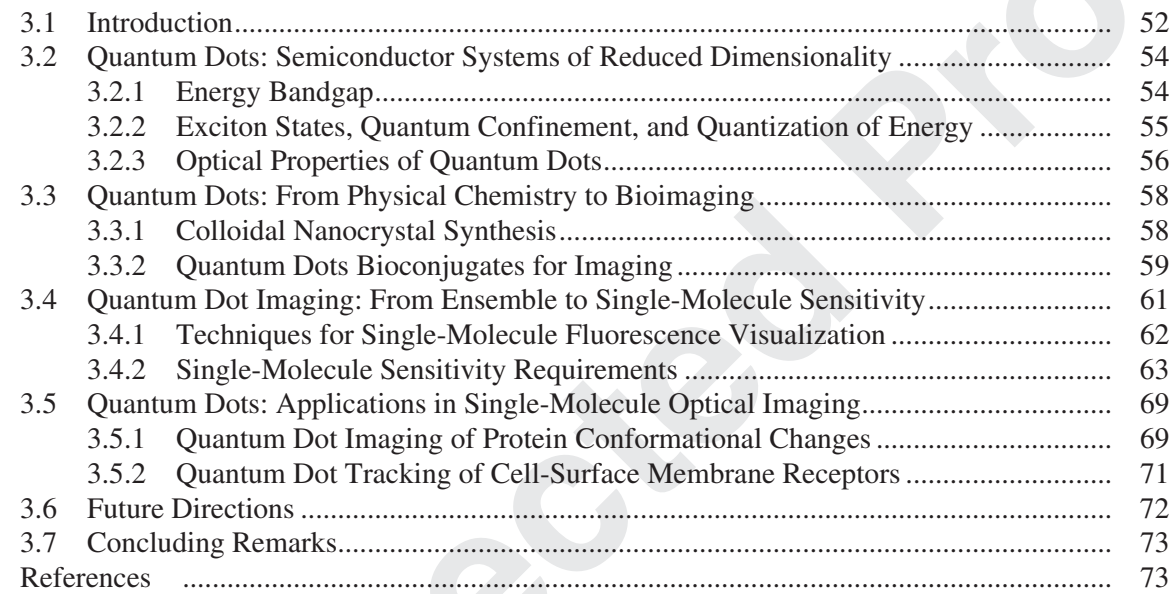

Abstract Coming from the electronic material sciences, semiconductor nanocrystals, called quantum dots (QDs), have emerged as new powerful fluorescent probes for in vitro and in vivo biological labeling and single-molecule experiments. QDs possess several unique optical properties that make them very attractive over conventional fluorescent dyes and genetically encoded proteins technologies. They have precise emission color tunability by size due to quantum confinement effects, better photostability and brightness, wide absorption band and very narrow emission band for multiplexing, and increased fluorescence lifetimes. These characteristics, combined with some dramatic progresses achieved in surface chemistry, biocompatibility and targeting strategies have allowed their recent advances in the field of single-molecule detection and imaging

Shimon Weiss

Department of Physiology, David Geffen School of Medicine, University of California at Los Angeles, Los Angeles, CA 90095-1569. United States of America

e-mail: sweiss@chem.ucla.edu 
using diverse microscope geometries like confocal microscopy, total internal reflection diverse microscopy or basic principes of QDs' electronic structure necessary to understand their fundamental optical and physical properties and goes on to present recent QDs' uses in biological imaging with an emphasis on single-molecule detection.

\subsection{Introduction}

In its visionary lecture delivered at Caltech in 1959, Richard Feynman suggested that "strange phenomena" would occur when a material's size is reduced to the

[Au1] nanometer scale (Feynman 1961). For semiconductor material, these radical changes occur when quantum-size phenomena (quantum confinement) take over conventional bulk properties. As the material's size is reduced in at least one dimension (in the 1 to $10 \mathrm{~nm}$ range), new material's properties start to emerge. These mesoscopic semiconductor structures of reduced dimensions (2-D quantum wells, 1-D quantum wires, and 0-D quantum dots) show unique size-dependent optical and electrical properties due to quantum confinement. Quantum dots (QD) are often dubbed "qdots," "quantum crystallites," or more simply "nanocrystals."

Predating our modern solid-state physics understanding of this phenomenon, centuries-old color-stained glass artworks decorating medieval cathedral windows are a vivid example of quantum confinement. These glass-melts contain colloidal particles made of zinc, cadmium sulfides, and selenides. It is only recently that the size-dependent optical properties of nanocrystals have been described within the framework of quantum confinement due to the pioneering work of the St. Petersburg school in Russia (Efros and Efros 1982; Ekimov and Onuschenko 1982; Ekimov et al. 1980) and the Bell Labs in the United States (Rossetti et al. 1983).

Nanocrystals of semiconductor material used in biological applications (e.g., CdSe, $\mathrm{PbS}$ ) are synthesized in solution using colloidal chemistry (Yin and Alivisatos 2005). They are in general spherical faceted crystals characterized by a diameter smaller than their exciton Bohr radius, a length scale that characterizes the typical electron-hole distance in the bulk material. The QDs small size (about 2 to $10 \mathrm{~nm}$ wide which corresponds to a few hundreds to a few thousands of atoms (Kadavanich et al. 2001)) imparts to them useful electronic and optical properties, very different from the properties of the bulk material on which they are based, most notably fluorescence. They behave as single "atomlike" quantum entities and adopt new properties that depend directly on their size (Alivisatos 1996a). Therefore, QDs are sometimes referred to as "artificial atoms" and even have their own periodic table (Ashoori 1996).

Their most useful property for imaging is the tunability of the absorption and emission spectra, which are related to the QD diameter, a parameter that can be precisely controlled by the duration of the nanocrystal growth. This simple control, as well as the availability of a variety of materials with significantly different properties allows for the synthesis of a continuous palette of emission colors spanning the soft ultraviolet (UV), the visible, and the near-infrared (NIR) portion of the light spectrum. These and several other unique QD optical properties have recently galvanized 
the biological sciences and include improved signal brightness, resistance against photobleaching, and simultaneous excitation of multiple fluorescence colors.

Since the first experiments demonstrating their potential uses as optical probes in biological imaging (Bruchez et al. 1998; Chan and Nie 1998), QDs have been applied in most biotechnological applications using fluorescence, including DNA array technology, immunofluorescence assays (reviewed in Alivisatos (2004)), and cell and animal biology (reviewed in Medintz et al. (2005) and Michalet et al. (2005)).

A survey of the recent literature testifies to the ever-growing interest of QDbased technologies in the biosciences. Publications using QD imaging probes in biological research have increased exponentially as robust QD sources became commercially available and readily formulated for the needs of the experimentalists. However, despite this current enthusiasm, QD technology will most likely not replace the well-established fluorescent dye technologies but rather will complement some of their shortcomings in niche applications (Michalet et al. 2005). For example, QDs emitting in the far-red and NIR regions of the spectrum could be of great utility for in vivo biological imaging, diagnostic, and possibly even therapeutics, due to their good separation from autofluorescence background and increased penetration of excitation and light emission through thick tissues.

Kim et al. (2004) have recently demonstrated in pigs and mice the superiority of NIR QDs in sentinel lymph node (SLN) mapping, a major procedure in breast cancer surgery. Because the QDs allowed image guidance throughout the procedure, virtually free of any background, the size of the incision that ensured the successful removal of the SLN was reduced. The imaging of SLN in real-time without the need of traditional dyes or radioactive tracers is a significant breakthrough that suggests that NIR imaging of QDs could possibly aid surgical procedures in humans (Kim et al. 2004). QDs are also proving to be excellent probes for two-photon confocal microscopy and deep-tissue imaging given their large absorption cross-section (Larson et al. 2003). But when combined with ultrasensitive microscopy techniques, QDs are showing their true potential by allowing visualization of cellular processes at a molecular scale (Dahan et al. 2003; Lidke et al. 2005; Warshaw et al. 2005).

Single-molecule microscopy is a novel high-resolution, high-sensitivity optical technique that is revealing a wealth of new information about the dynamics of isolated molecules, in vitro and in their cellular environment (Harms et al. 2001; Iino et al. 2001; Schutz et al. 2000; Seisenberger et al. 2001; Ueda et al. 2001). The ability to watch one molecule at a time can unravel subtle and discrete subpopulations and/or rare events that would otherwise be hidden in heterogeneous samples because of the statistical and temporal averaging from which those ensemble measurements suffer (Michalet et al. 2003). Because of their unmatched photostability, QDs are the probes of choice for long observation of individual cellular proteins, reconciling the inherent incompatibility in time scales of organic dyes' photobleaching lifetime and desired observation time (Weiss 1999). Additionally, single-molecule fluorescence microscopy can be combined with electron microscopy (EM) given QDs high electronic density as it has been demonstrated recently (Dahan et al. 2003). Thus, QDs have the unique potential to provide spatial, temporal, and structural information at all length scales: from the whole-body down to nanometer resolution with a single probe. 
This chapter begins by setting out the basic principles of QDs' electronic structure necessary to understand their fundamental optical and physical properties. It then gives a brief review of the dramatic progress achieved in surface chemistry, biocompatibility, and targeting strategies that allowed their recent advances in imaging applications. It then goes on to present recent QDs' uses in biological imaging with an emphasis on single-molecule detection. QD in vitro assay labeling and sensing are discussed elsewhere (Alivisatos 2004; Medintz et al. 2005; Michalet et al. 2005). Finally, it outlines the future directions and potentials of QD technology in fluorescence spectroscopy of single-biomolecules.

\subsection{Quantum Dots: Semiconductor Systems of Reduced Dimensionality}

This section outlines the essential concepts of low-dimensional semiconductor structures and provides a framework to understand why QDs demonstrate desirable optical properties for imaging. Interested readers should consult textbooks on quantum mechanics, solid-state physics, or QDs (Atkins and Friedman 1997; Gaponenko 1998; Kittel 1996) for an in-depth discussion.

\subsubsection{Energy Bandgap}

Electrons in atoms and molecules have defined energies, described by quantum mechanics as "energy levels". Only two electrons can exist in any one energy level (Pauli exclusion principle) with the consequence that electrons will start filling energy levels from the lowest energies upwards. In crystalline solids (such as metals, semiconductors, and insulators), however, electrons are arranged in a near continuum of energy levels known as energy bands due to the overlap of their atomic orbitals in the closely packed crystalline arrangements (Kittel 1996).

The highest filled band at $T=0 \mathrm{~K}$ is known as the valence band and the first unfilled band above the valence band is referred to as the conduction band. Regions in energy for which no wavelike electron orbitals exist separate valence and con-

[Au2] duction bands. Such forbidden regions are called energy gaps $\left(E_{g}\right)$ or band gaps, and result from the interaction of the conduction electron wavefunctions with the ion cores of the crystal.

The band theory of materials explains qualitatively the difference among conductors, semiconductors, and insulators. In conductors (e.g., metals), valence and conduction bands overlap $\left(E_{g}=0\right)$, allowing free electrons to participate in the conduction process. To the contrary, bulk semiconductors have a filled valence band and a mostly empty conduction band separated by an $E_{g}$ typically of about 1-2 electron volt $(\mathrm{eV})(3.8 \mathrm{e}-23$ to $7.6 \mathrm{e}-23 \mathrm{kcal})$. Insulators are not different from semiconductors except for the fact that in insulators, $E_{g}$ is far greater than the thermal energy of the electron $\left(E_{g}>2 \mathrm{eV}\right.$, Figure 3.1). 

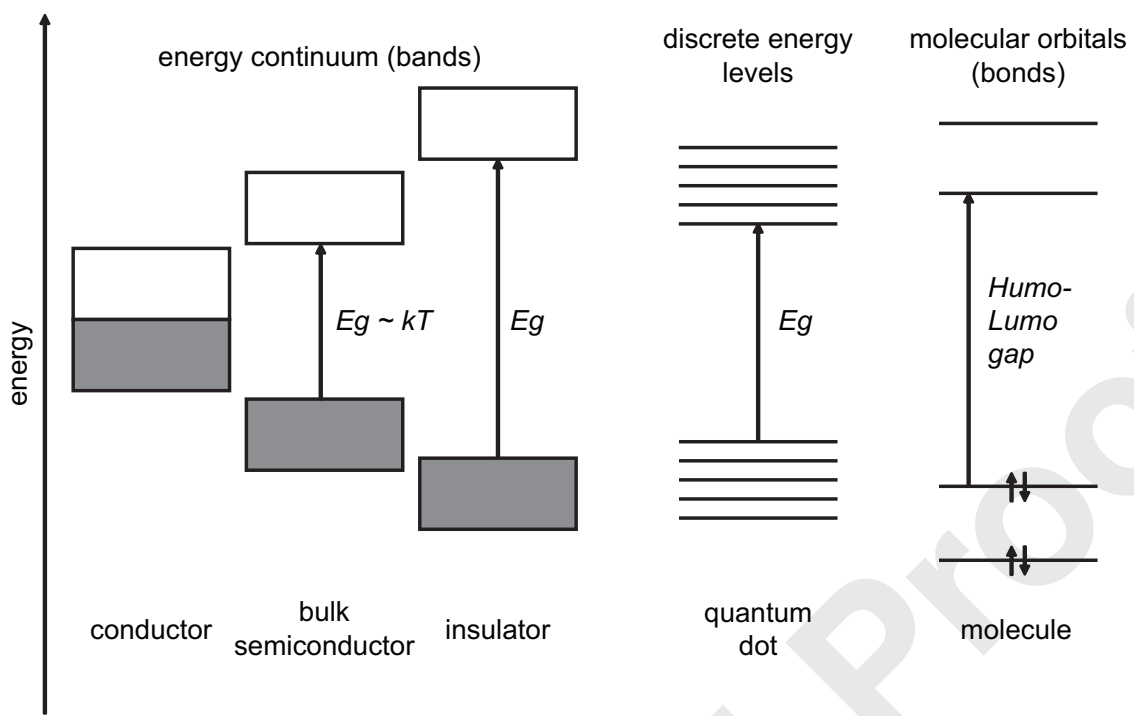

Figure 3.1 Simplified energy level diagram for crystalline inorganic solids and molecules. Grey boxes: filled valence bands; empty boxes: empty conductive bands (at $0 \mathrm{~K}$ ). The vertical arrow represents the bandgap energy Eg for the solids, and the highest occupied molecular orbitallowest unoccupied molecular orbital (HOMO-LUMO) energy gap in the molecule. The electronic structure of semiconductor QD is in the intermediate regime between bands and bonds. (Adapted from Murphy (2002) and Kittel (1996))

\subsubsection{Exciton States, Quantum Confinement, and Quantization of Energy}

Upon photon absorption of light with energy $h v>E_{g}$, an electron is promoted from the filled valence band to the (mostly) empty conduction band, leaving a positively charged vacancy, or "hole," which can be described as a quasiparticle with a mass, momentum, and energy interacting with its surrounding. The excited electron in the conduction band and the hole in the valence band are of opposite signs and therefore interact with each other via Coulomb interaction, similarly to the proton and electron of a hydrogen atom. As in the hydrogen atom, the distance separating the electron-hole pair (such a bound pair is referred to as an "exciton") is described by the Bohr radius $\left(a_{\mathrm{ex}}\right)$ and is characteristic of the material under consideration:

$$
a_{e x}=\varepsilon h^{2} / \pi m_{e x} e^{2}
$$

where $\varepsilon$ is the semiconductor permittivity, $m_{\mathrm{ex}}$ is the reduced exciton mass (as determined by ion cyclotron resonance (Gaponenko 1998; Hannay 1959; Mahan 2000) ), $h$ is Planck's constant, and $e$ the electron charge. The exciton's Bohr radius is 
typically about 1 to $10 \mathrm{~nm}$ (Gaponenko 1998) for most semiconductors (e.g., $a_{\mathrm{CuCl}}$ $\sim 7 \AA, a_{\mathrm{GaAs}} \sim 100 \AA$, and $\left.a_{\mathrm{CdSe}} \sim 56 \AA\right)$.

For semiconductor dimensions smaller than the exciton's Bohr radius, the motion of the excitons becomes obviously confined in space. Confinement in one dimension gives rise to quantum wells, in two dimensions to quantum wires and when all three spatial dimensions are confined, a quasi-zero-dimensional dot (quantum dot) structure is produced (Alivisatos 1996a,b; Empedocles and Bawendi 1999; Steigerwald and Brus 1990; Weller 1993a,b, 1998; Zhang 1997). The confined exciton behaves as a particle trapped in a spherical three-dimensional potential box (i.e., classical particle-in-a-box problem). Practically, calculations and experiments show that the three-dimensional reduction of the QD nanocrystal dimensions results in the appearance of discrete energy levels at the edges of the conduction and valence bands reminiscent of that of atoms or that of the familiar HOMO-LUMO-energy gap for small molecules (Figure 3.1). QD nanocrystals therefore exhibit a mixture of properties in this mesoscopic regime, transitioning from bulk semiconductor (i.e., with energy bands) properties to single atomlike properties (i.e., with discrete energy levels). QDs are thus also referred to as "artificial atoms" or "hyperatoms".

\subsubsection{Optical Properties of Quantum Dots}

\subsubsection{Tunable Bandgap and Size-Dependent Photoluminescence}

The separation of the last discrete electronic level of the valence band and the first discrete level of the conduction band, or first exciton energy is related to the bulk bandgap energy $E_{g}$ and the size of the QD in such a way that $E_{g}$ increases as the dimensions of the particle decreases (Alivisatos 1996a,b; Brus 1984; Weller 1998; Zhang 1997). This first exciton energy of a nanocrystal is often referred to as its bandgap energy, and this is the terminology that is used henceforth.

Because particle size directly relates to the QD's bandgap energy, QDs have sizeand composition-dependentabsorption andemission(Figure3.2A). Photoluminescence in QDs is essentially described in terms of the annihilation of the exciton by radiative recombination. Therefore the emission wavelength follows the size-dependent changes in the bandgap; that is, the smaller the QD, the bluer is its emission (tunable emission). In practice, it is possible to continuously tune the spectroscopic properties of QDs by precisely controlling the duration, temperature, and ligand molecules used during the colloidal chemical synthesis (Alivisatos 1996a).

As in the case of bulk semiconductors, the QD's absorption has an increased probability at higher energies (i.e., shorter wavelength), and is characterized by a broadband absorption spectrum, in marked difference with standard fluorophores (Figure 3.2B). Also, the ensuing ensemble emission spectrum is slightly Stokes-shifted with respect to the excitation band edge and consists of a narrow and symmetric Lorentzian band (30-40 nm full-width-at-half maximum (FWHM) or less) in sharp contrast with 
the red-tailed emission spectra of most fluorophores (Figure 3.2B). The width of the ensemble spectrum is due to both the size dispersion of the QD particles inherent to their synthesis (as we discuss in Section 3.3.1) and spectral jumps caused by local changes in the QD's surface. Spectral jumps are another illustration of the atomlike nature of QDs and their similarities to molecules, for which similar phenomenon have been described (Ambrose and Moerner 1991; Orrit and Bernard 1990).

\subsubsection{Bandgap and Wavefunction Engineering}

The fate of the photo-generated electron-hole pair is influenced by unpassivated surface defects in the crystal structure, which can act as temporary "traps" for the electron or hole, preventing their radiative recombination. The alternation of trapping and untrapping events results in intermittent fluorescence, or blinking typical of QDs, visible at the single-molecule level (Nirmal et al. 1996) and reduces the overall quantum yield (QY), defined as the ratio of emitted to absorbed photons.

One way to eliminate these "trap" states and protect surface atoms from photooxidation and other chemical reactions is to grow a few atomic layers thick protec-
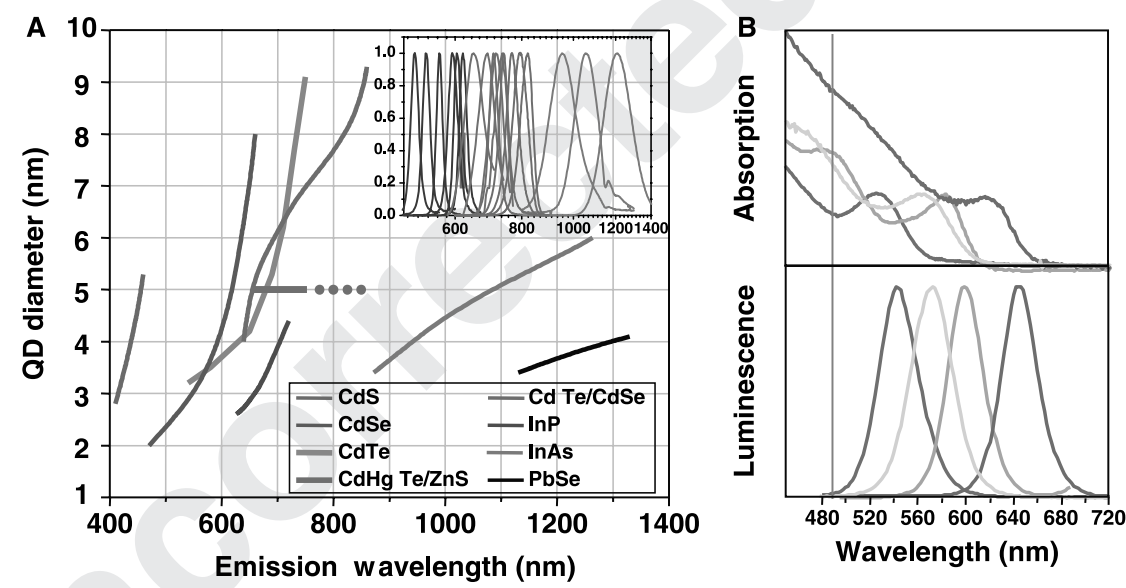

Figure 3.2 (A) Emission maxima and sizes of quantum dots of different composition. QDs can be synthesized from various types of semiconductor materials (II-VI: CdS, CdSe, CdTe...; III-V: InP, InAs...; IV-VI: PbSe...) characterized by different bulk bandgap energies. The curves represent experimental data from the literature on the dependence of peak emission wavelength on QD diameter. The range of emission wavelength is 400 to $1350 \mathrm{~nm}$, with size varying from 2 to $9.5 \mathrm{~nm}$ (organic passivation/solubilization layer not included). All spectra are typically around 30 to $50 \mathrm{~nm}$ (FWHM). Inset: Representative emission spectra for some materials. (B) Absorption (upper curves) and emission (lower curves) spectra of four $\mathrm{CdSe} / \mathrm{ZnS}$ QD samples. The blue vertical line indicates the $488 \mathrm{~nm}$ line of an argon-ion laser, which can be used to efficiently excite all four types of QDs simultaneously. ((A) Reprinted with permission from Michalet et al. (2005). Copyright 2005 AAAS. (B) Reprinted with permission from Gerion et al. (2001). Copyright 2001 American Chemical Society) 
tive shell of a larger bandgap material and similar crystalline mesh on top of the nanocrystal core (Dabbousi et al. 1997; Eychmüller et al. 1993; Kortan et al. 1990; Mews et al. 1994; Peng et al. 1997). This "shell" layer can increase the QY to values up to $\sim 90 \%$ (Reiss et al. 2002) by eliminating surface traps and better confining the exciton into the core. This step also enhances QDs' photostability by several orders of magnitude relative to conventional dyes (Sukhanova et al. 2004).

A typical example is a $\mathrm{ZnS}$ shell $\left(E_{\mathrm{g}}=3.8 \mathrm{eV}\right.$ corresponding to absorption onsets at $\sim 325 \mathrm{~nm}$ (Dabbousi et al. 1997, Hines and Guyot-Sionnest 1996) ) used to cap CdSe cores $\left(E_{g}=1.7 \mathrm{eV} \sim 720 \mathrm{~nm}\right.$; Murphy (2002) in a so-called band alignment of type-I. CdS $\left(E_{g}=2.4 \mathrm{eV} \sim 520 \mathrm{~nm}\right.$; Peng et al. (1997) ) is better lattice-matched to $\mathrm{CdSe}$, but ZnS has a larger band offset (Böer 1990) resulting in better confinement. Leakage of the wavefunction into the shell results in a slightly red-shifted spectra of the "core-shell" structure as compared to the core alone (Peng et al. 1997).

Recently, atomic layer-by-layer successive shell deposition has revealed systematic changes of lifetime with shell growth of type-II CdTe/CdSe QDs ( $\mathrm{Li}$ et al. 2005). This demonstrates the high-level of wavefunction manipulation and radiative recombination control that can be achieve in the quantum size regime.

One can take advantage of the long QDs' lifetime (>10ns; Efros and Rosen (2000) to separate their signal from background autofluorescence encountered in cells by timegated imaging detection (Dahan et al. 2001) as discussed in Sections 3.4 and 3.6.

\subsection{Quantum Dots: From Physical Chemistry to Bioimaging}

QDs have been obtained using various technologies; from "top-down" techniques by means of reducing a quantum wells in the two lateral dimensions to "bottom-up" precision growth techniques borrowed from glass technology and colloidal chemistry (Gaponenko 1998). Here, we provide a brief overview of the state-ofthe-art fabrication of fluorescent colloidal QDs, a class of QDs exclusively used for biological labeling.

\subsubsection{Colloidal Nanocrystal Synthesis}

"Bottom-up" approaches allow the most precise growth control of the QDs, providing very narrow size distributions ( $<5 \%$ standard deviation in diameter) necessary for imaging applications. In the colloidal approach, QDs are grown in a solvent solution. Molecular or ionic QD precursors are reacted at high temperature $\left(300^{\circ} \mathrm{C}\right)$ in a single vessel, together with a stabilizing agent (an organic surfactant) that stabilizes the growing particles. Typically, a mixture of surfactants such as trioctylphosphine oxide (TOPO) and trioctylphosphine (TOP) is used to coat the growing crystallite cores and prevent their aggregation, as well as the dissolution of smaller nanocrystals in favor of larger ones (a process known as Ostwald ripening). 
Murray, Norris, and Bawendi (1993) first introduced this concept for the growth of CdSe nanocrystals in TOPO.

Size tuning (from 2.0 to $8.0 \mathrm{~nm}$ ) is precisely controlled by factors such as the duration, temperature, relative concentration, and rate of addition of ligand molecules (Alivisatos 1996a; Yin and Alivisatos 2005) and is monitored by absorption and photoluminescence spectra of aliquots taken along the time-course of the reaction (Bowen Katari et al. 1994). Cooling down the reaction vial eventually stops the growth altogether. QD cores are then purified through successive "size-selective precipitation" and washing steps, which further improve their size distribution (Murray et al. 1993; Vossmeyer et al. 1994). They are kept in nonpolar solvents such as butanol or toluene. High-resolution TEM indicates that the cores obtained by these methods are made of a few hundred to a few thousand atoms (Kadavanich et al. 2001; Murray et al. 1993). The QY of the resulting core is generally modest (a few percent only) until an outer shell of higher bandgap material is added (see below).

\subsubsection{Quantum Dots Bioconjugates for Imaging}

The use of semiconductor QDs as biological fluorescent probes requires that they: (i) are water soluble; (ii) offer reactive groups on their surface for subsequent bioconjugation; (iii) maintain all their photophysical properties when transferred into aqueous buffers (wide excitation band, narrow emission, long fluorescence lifetime, high quantum yield, high photochemical and photophysical stability); (iv) remain monodispersed; and (v) have a reasonable final small size. Therefore, continuing research efforts focus on further developing and improving the organicinorganic interface.

\subsubsection{Quantum Dot Solubilization}

Inasmuch as QDs are mostly synthesized in nonpolar organic solvents, their hydrophobic surface must be converted to a hydrophilic one in order to solubilize them in aqueous buffers. This can be achieved by either surfactant exchange, a process primarily driven by mass-action in which the native TOP/TOPO is substituted with bifunctional ligands or by insulation of the original hydrophobic QD within a heterofunctional amphiphilic coating (Figure 3.3).

These various QD solubilization strategies include: (i) ligand exchange with simple thiol-containing molecules (Chan and Nie 1998; Pathak et al. 2001) or more sophisticated ones such as oligomeric phosphines (Kim and Bawendi 2003), dendrons (Guo et al. 2003), and peptides (Akerman et al. 2002; Pinaud et al. 2004); (ii) encapsulation by a layer of amphiphilic diblock (Wu et al. 2003), or triblock copolymers (Gao et al. 2004), in silica shells (Bruchez et al. 1998; Gerion et al. 2001), phospholipid micelles (Dubertret et al. 2002), polymer beads (Gao et al. 2002), polymer shells (Pellegrino et al. 2004), amphiphilic polysaccharides (Osaki et al. 2004); and (iii) combination of 


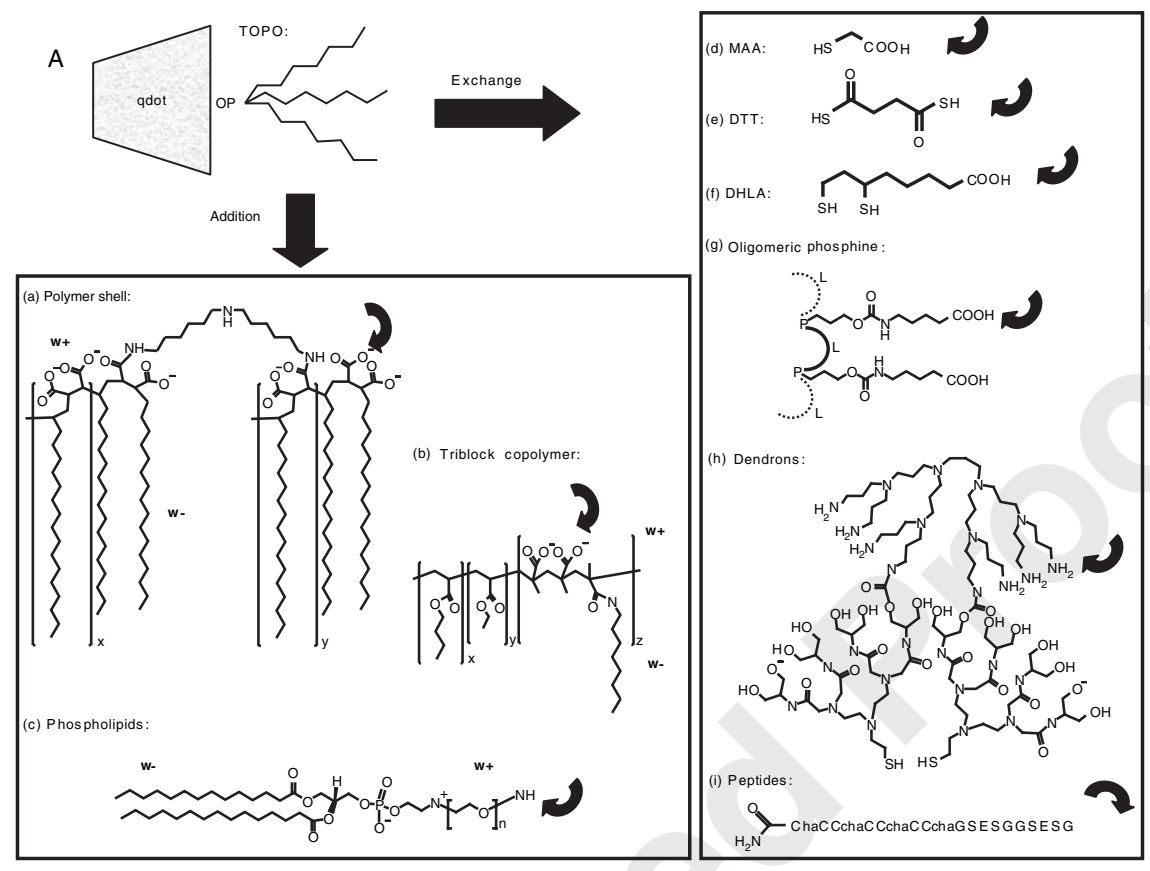

Figure 3.3 Quantum dot solubilization and functionalization. (A) Surface chemistries. TOPO (trioctylphosphine oxide)-passivated QDs can be solubilized in aqueous buffer by addition of a layer of amphiphilic molecules containing hydrophilic $(\mathrm{w}+)$ and hydrophobic $(\mathrm{w}-)$ moieties, or by exchange of TOPO with molecules that have a $\mathrm{Zn}$-coordinating end (usually a thiol group, $\mathrm{SH}$ ) and a hydrophilic end. Examples of addition include (a) formation of a cross-linked polymer shell, (b) coating with a layer of amphiphilic triblock copolymer, and (c) encapsulation in phospholipid micelles. Examples of exchange include (d) mercaptoacetic acid (MAA), (e) dithiothreitol (DTT), (f) dihydrolipoic acid (DHLA), (g) oligomeric phosphines, (h) cross-linked dendrons, and (i) peptides. The curved arrow indicates sites available for further functionalization. (Reprinted with permission from Michalet et al. (2005). Copyright 2005 AAAS)

layers of different molecules conferring the required colloidal stability to QDs (Mattoussi et al. 2000; Sukhanova et al. 2004). Recently, some groups have developed promising water-based synthesis (Gaponik et al. 2002; Rogach et al. 2001) yielding particles emitting from the visible to the NIR spectrum that are intrinsically watersoluble, but have yet to be tested in biological environments.

\subsubsection{Quantum Dot Functionalization}

Surface functionalization with hydrophilic ligands serves as a point of chemical attachment for biomolecules. QD ligands containing either an amine or a carboxyl group offer the possibility to cross-link molecules containing a thiol group (Akerman et al. 2002; Dubertret et al. 2002; Mitchell et al. 1999) or an $N$-hydroxysuccinimyl-ester moiety (Bruchez et al. 1998; Pinaud et al. 2004) using standard 
bioconjugation reactions. Another approach uses electrostatic interactions between QDs and charged adapter molecules, or proteins modified to incorporate charged domains (Goldman et al. 2002). These functionalization steps can be repeated to add or change functionality. For instance, streptavidin-coated QDs can be used in combination with biotinylated proteins, antibodies, or nucleic acids (Bentolila and Weiss 2006; Chan et al. 2005; Dahan et al. 2003; Jaiswal et al. 2003; Mansson et al. 2004; Pinaud et al. 2004; Wu et al. 2003). By extension, a generic three-layer approach using (i) an antibody against a specific target, (ii) a biotinylated secondary antibody against the first, and (iii) a streptavidin-coated QD, allows QD labeling of most types of target (Dahan et al. 2003; Wu et al. 2003). However, the "sandwich" approach results in large structures that may impede further use of QDs in some applications.

To circumvent that problem, our laboratory has developed a unique peptide-based solubilization and functionalization approach that maintains the small size of the particles. This is achieved by exchanging the surfactants with synthetic phytochelatinlike $\alpha$-peptides (Pinaud et al. 2004) that mimic the natural formation of peptide-coated QDs during heavy metal detoxification processes in plants and yeasts (Cobbett 2001; Dameron et al. 1989; Dameron and Winge 1990a,b; Stillman 1995). These peptides comprise a metal-chelating and hydrophobic domain ensuring binding to the QD surface, and a hydrophilic tail that provide solubilization and stability in buffers (Figure 3.3i). A single binding domain of the peptide containing cysteines (C) and hydrophobic unnatural amino acids 3-cyclohexylalanines (Cha) is responsible for surface recognition and attachment to QDs, whereas the variable hydrophilic domain provides solubilization and functional groups for bioconjugation. This surface chemistry is very simple and achieved in a single reaction step. The resulting particles have excellent colloidal and photophysical properties (Doose et al. 2005, Pinaud et al. 2004) suitable for single-molecule techniques, as we discuss in Section 3.4. It is important to note that our peptide-coating approach maintains the overall size of the QDs relatively small ( 8 to $13 \mathrm{~nm}$ ) as compared to $\sim 18 \mathrm{~nm}$ for phospholipid-coated QDs, and $30 \mathrm{~nm}$ for commercial nanorods protected by a polymer coat (Doose et al. 2005).

\subsection{Quantum Dot Imaging: From Ensemble to Single-Molecule Sensitivity}

The successful developments of a wide range of methods for bioconjugating fluorescent colloidal QDs have accelerated their use in biological imaging and ultrasensitive detection in vitro applications, in live cells and in whole organisms (reviewed in Alivisatos (2004), Medintz et al. (2005), and Michalet et al. (2005)). Most appealing for the biosciences, are the high brightness, high resistance to photobleaching, and the ability to size-tune fluorescent emission of these inorganic-biological hybrid nanostructures. When combined with ultrasensitive optical techniques the enhanced photophysical properties of QDs fulfill the stringent requirements needed to achieve single-molecule sensitivity to study complex 
dynamic cellular processes. In the following, we discuss the experimental layouts and the physical observables of these microscopy techniques.

\subsubsection{Techniques for Single-Molecule Fluorescence Visualization}

The first single protein visualized by fluorescence microscopy was an antibody molecule labeled with about 100 fluorophores (Hirschfeld 1976). Since then, progress in molecular imaging has advanced the boundary of detection down to the single-molecule-single-fluorophore complexes in their native cellular environment (Harms et al. 2001; Iino et al. 2001; Schutz et al. 2000; Seisenberger et al. 2001; Ueda et al. 2001). These advances have been critically dependent upon three main factors: (i) the introduction of enhanced indicator probes (new variants of genetically encoded fluorescent proteins, novel inorganic nanomaterials and novel organic fluorophores); (ii) the innovation of new optics and detectors allowing steady gain in microscope collection optics, detector sensitivity and dynamic range; and (iii) the enhanced computer capabilities to process an ever-growing flow of data.

Single-molecule fluorescence imaging techniques rely mainly on two schemes: wide-field excitation/detection and point excitation/detection geometries (Figure 3.4). The wide-field format uses epifluorescence illumination with high-power arc lamps (e.g., mercury) in a Kohler-illumination arrangement (Goulian and Simon 2000) or defocused laser excitation (Schmidt et al. 1996), or total internal reflection excitation (TIR; Axelrod 2003; Funatsu et al. 1995) and a wide area detector (camera).

TIR differs from the first two wide-field methods in limiting the excitation light to the "evanescent field" region at the boundary between the coverslip and water (or

(a) epi.

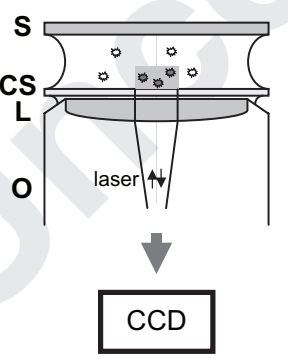

(b) TIR (obj.)

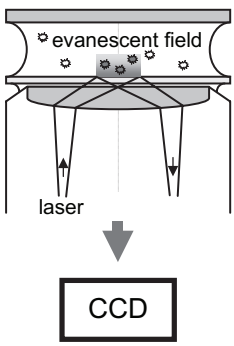

(c) TIR (prism)

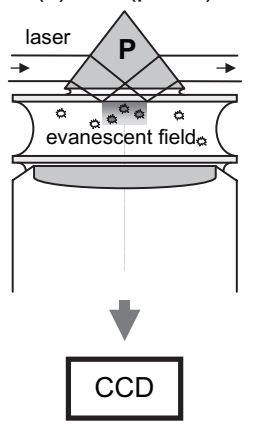

(d) Confocal

Figure 3.4 Different excitation/detection geometries utilized in single-molecule microscopy: (a) epifluorescence, (b) through-the-objective TIR, (c) prism TIR, and (d) confocal microscopy (laser scanning beam or scanning stage). S: slide; CS: coverslip; L: lens; O: objective, typically high NA oil-immersion (>1.4 for TIR); P: prism. Area (CCD) or point (PMT or APD) detectors are shown. The filled and empty circles represent fluorescent molecules that are visible and invisible, respectively. Figure is not to scale. (Adapted from Moerner (2003) and Kellermayer (2005)) 
air) (Figures 3.4b and c). The intensity of the evanescent field decays exponentially as the distance from the boundary increases, thus limiting the excitation depth to a very narrow layer, typically one hundred to a few hundreds of nanometers (Axelrod 2003). Because TIR is a proximity imaging method its application is limited to molecules immobilized on the surface, molecules diffusing close to the surface, or cell surface (the plasma membrane) near the solid support.

The large area detectors used with wide-field excitation geometries include cooled back-thinned CCD cameras, intensified CCD cameras (ICCD; for instance SIT cameras), or electron-multiplying CCD cameras (EMCCD), with detection quantum efficiencies (QE) ranging from 40 to $90 \%$ in the visible spectral range. Such detectors allow the simultaneous observation of multiple single molecules scattered over a relatively large field of view (Goulian and Simon 2000; Michalet et al. 2003; Schmidt et al. 1996).

In recent years, single QDs have been successfully observed and tracked over extended period of times (up to a few hours) using TIR microscopy

[Au3] (Michalet et al. 2001) or basic wide-field epifluorescence microscopy (Figure 3.5 and Dahan et al. (2003), Hohng and Ha (2004), Lidke et al. (2005), and Warshaw et al. (2005)).

The point excitation/detection scheme is based on a confocal laser scanning or stage scanning microscopy (CLSM or CSSM, respectively). The laser beam is

[Au4] focused through an objective lens onto the specimen (Pawley 1995; Webb 1999). The diffraction-limited focal spot defines the excitation volume, which is on the order of a few femtoliters $\left(10^{-15} \mathrm{l}\right)$. In CLSM, the laser beam is raster-scanned across the field of view and the image is acquired pixel by pixel using a point-detector (typically a photomultiplier tube of $\mathrm{QE}<20 \%$ ) and reconstructed by software. This scheme, however, provides relatively poor sensitivity. In CSSM, the excitation beam is stationary on the optical axis and the sample-stage is raster-scanned across the field of view to produce the pixel-by-pixel image.

Ultrasensitive avalanche photodiode point detectors (APD, QE $<90 \%$ ) are typically used for this scheme, which is usually slower and therefore adapted to

[Au5] very low light level imaging (Michalet et al. 2001). The CSSM approach is suited for the acquisition of fluorescence time traces of immobilized molecules (Macklin et al. 1996) or isolated QDs (Lacoste et al. 2000) and provides high spatial and temporal resolution, as well as access to fluorescence lifetime information but is inadequate to simultaneously study dynamic processes of many molecules. Widefield methods are therefore necessary for the high-throughput accumulation of a statistically significant number of single molecule trajectories (Funatsu et al. 1995; Schmidt et al. 1996).

\subsubsection{Single-Molecule Sensitivity Requirements}

\subsubsection{Signal and Noise}

A prerequisite to achieving single-molecule sensitivity is a careful optimization of background, signal, and noise emission which are manifested in the signal-to-noise 

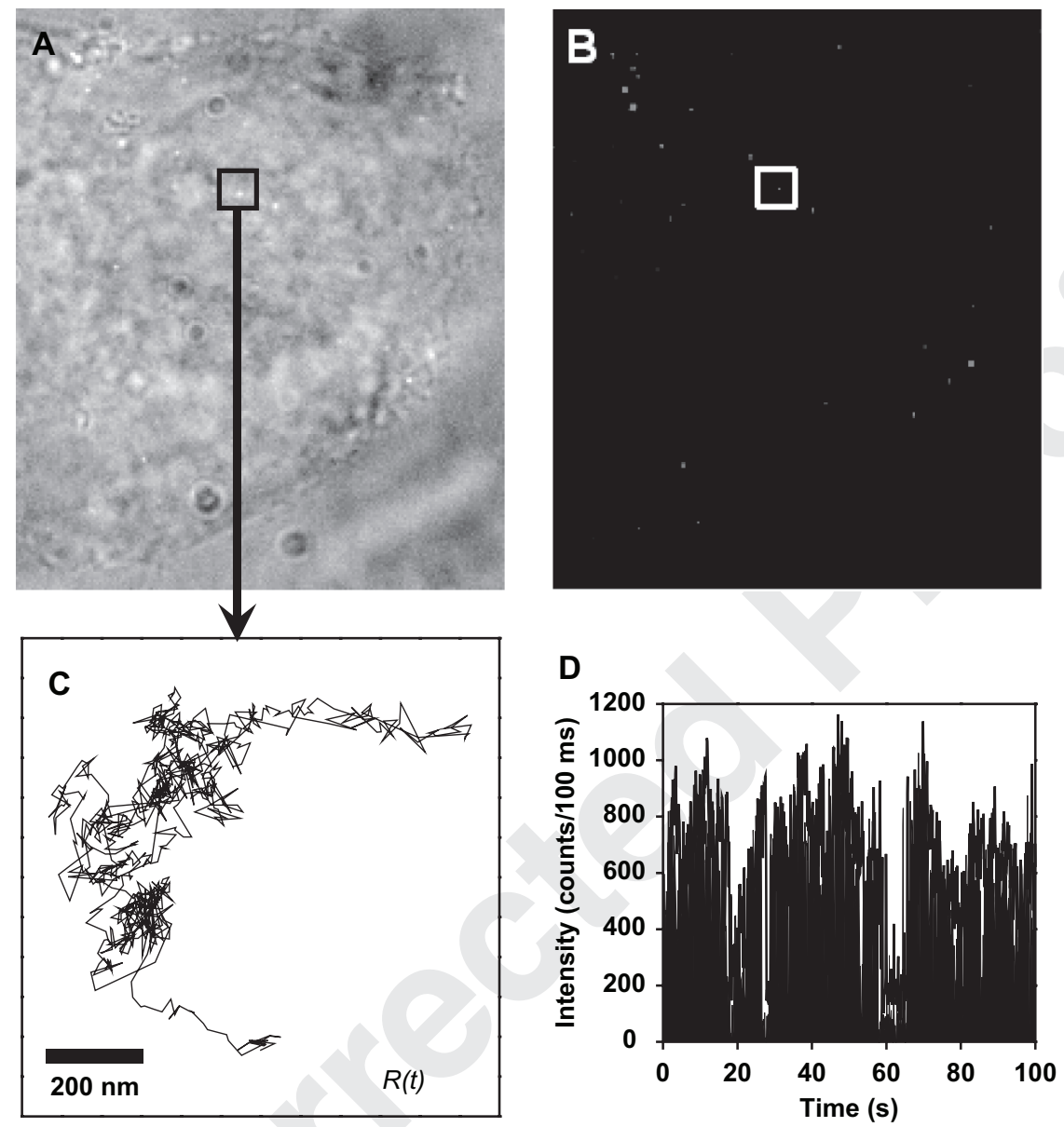

Figure 3.5 Single-particle tracking in a live-cell. The brightness and photostability of QDs permits single QD observation over a long period of time. Live HeLa cells stably transfected with a plasmid expressing a chimeric avidin-CD14 receptor were grown on fibronectin-coated glass coverslips, incubated with biotin-QDs (emission: $630 \mathrm{~nm}$ ), and washed with the observation medium. The cells were observed in DIC (A) and epifluorescence (B) on an inverted microscope (Axiovert 100, Zeiss) using a simple Hg lamp and imaged with a cooled monochrome CCD camera (CoolSnap HQ, Roper Scientific). Single-QDs were observed to diffuse at characteristically different rates in different regions of the membrane or inside the cytosol (data not shown). C: The 1000 steps (100 ms/step) trajectory, $\mathrm{R}(\mathrm{t})$, of the QD localized in the region marked in panel $(\mathbf{A}, \mathbf{B})$, with (D) the corresponding QD intensity, $\mathrm{I}(\mathrm{t})$. The blinking pattern (succession of on and off emission) demonstrates that a single QD was observed. (Reprinted with permission from Michalet et al. (2005). Copyright 2005 AAAS)

ratio (SNR) and the signal-to-background ratio (SBR) (Michalet et al. 2003; Paige et al. 2001)) defined as

$$
S N R=\frac{\text { Signal }}{R M S(\text { Signal }+ \text { Background }+ \text { electronic Noise })}
$$


and

$$
S B R=\frac{\text { Signal }}{\text { Background }} .
$$

On the instrument side, improving the collection efficiency can increase both SNR and SBR whereas using a higher excitation power and longer integration time will only improve the SNR. SBR can be increased by reducing the excitation volume through combination of focusing (as in CLSM) and/or specialized optical excitation (as with the evanescent field in TIR; Michalet et al. (2003), Paige et al. (2001)).

On the probe (fluorophore) side, desirable properties include high extinction (absorption) coefficient, high fluorescence QY, minimal intersystem crossing to triplet state, and high photostability (Kapanidis and Weiss 2002). QDs indeed fulfill these requirements.

QDs' high molar extinction coefficient $(\varepsilon)$, on the order of $10^{6} \mathrm{M}^{-1} 1 \mathrm{~cm}^{-1}$ in the visible, is $\sim 10-100$ times larger than that of standard organic dyes or genetically encoded fluorescent protein (Dabbousi et al. 1997; Leatherdale et al. 2002) and is the prime contributor to QDs' high SNR.

$\varepsilon$ directly relates to the number of photons $N$ (i.e., the "signal" in Equations (3.2) and (3.3) ) emitted from a single QD in a given acquisition time $\tau$ as follows,

$$
N=\alpha q \cdot\left(2.303 \varepsilon / N_{A}\right)(I \lambda / h c) \tau
$$

where $\alpha$ is the detection efficiency $(\sim 0.1-5 \%), q$ the emission quantum yield (30$80 \%), \lambda$ the QD emission wavelength, $h$ the Planck constant $\left(h=6.63 \times 10^{-34} \mathrm{~J} \mathrm{~s}\right), c$ the speed of light, $I$ the excitation power per unit area, and $N_{A}$ the Avogadro number.

Practically, this means that at comparable quantum yields and similar emission saturation levels (Doose et al. 2005), QDs are much brighter than dyes. In addition, because they are very photostable, they emit many more photons per particle prior to photobleaching compared to organic dyes (Ballou et al. 2004; Dubertret et al. 2002). These properties allow the detection of QDs' fluorescent signal way above background with the ultimate high sensitivity of one QD per target molecule (Figures 3.5, 3.8, Dahan et al. (2003), and Lidke et al. (2005)).

\subsubsection{Resolution and Localization}

An important issue in the quantitative data analysis of single-molecule microscopy/ spectroscopy relates to the accuracy with which the position of a single molecule can be determined.

In wide-field microscopy, TIR or CLSM, the ability to detect single molecules depends on those molecules being spaced far enough apart from each other (which means working at low dilution, usually within the ten to hundred picomolar concentration range). This is due to the fact that in light microscopy, spatial resolution (the ability to distinguish identical fluorescent point-sources at high density) is 
fundamentally limited by the diffraction of light. Indeed, the focusing of light produces a finite-size focal volume of about $250-300 \mathrm{~nm}$ in $x-y$ and $700-800 \mathrm{~nm}$ in $z$ in the visible range (Abbe 1873). This volume is mathematically described by dif-

[Au6] fraction theory and is called the point-spread-function (PSF; Pawley (1995)).

At the traverse focal plane $(x-y)$ the full-width-at-half-maximum (FWHM) of the focal spot is:

$$
\Delta x, \Delta y=\frac{\lambda}{2 \sin \alpha}
$$

and along the optical axis $(z)$ is:

$$
\Delta z=\frac{2 \lambda}{n \sin ^{2} \alpha}
$$

where $\lambda$ is the excitation wavelength, $n$ the refractive index, and $\alpha$ the semiaperture angle of the objective lens. It is customary to use the FWHM of the PSF as a direct measure of the resolution (Rayleigh criterion). Without resorting to super-resolution techniques (as we discuss later) fluorescent microscopes can thus resolve at best, $\lambda / 2 \sim 200 \mathrm{~nm}$ at the focal $(x-y)$ plane and only 500-800 $\mathrm{nm}$ along the optic axis $(z)$

[Au7] with visible light (Pawley 1995). As an example, in order to resolve two individual and identical QDs that emit $630 \mathrm{~nm}$ light, they need to be at least $240 \mathrm{~nm}$ apart in the $x-y$ plane when imaged with an oil-immersion objective with a numerical aperture (NA) of 1.3. This means that spatial resolution is first intrinsically limited by wavelength not by molecule concentration. Therefore, resolution must be confused neither with single-molecule sensitivity (Weiss 1999) nor with positioning accuracy and distance measurement between two spectrally distinct singlefluorescent molecules (Lacoste et al. 2000).

In fact, the localization of a fluorescent point-source (i.e., the center of the PSF) can be determined with much better precision (in a sense overcoming the diffraction limit) without improving the imaging resolution by fitting the PSF with a twodimensional Gaussian curve or using centroid-finding algorithms (Gelles et al. 1988), if a sufficient number of photons $N$ can be collected from the spot (Bobroff

[Au8] 1986, Michalet et al. 2001; Snyder et al. 2004, Yildiz et al. 2003). In the case of QDs, the high number of emitted (and collected) photons $N$ affords high localization accuracy. With signal high enough to overcome background, Equation (3.2) can be approximated by:

$$
S N R \approx \frac{\text { Signal }}{R M S(\text { Signal })}
$$

The localization precision is solely limited by the SNR as calculated by many authors (Bobroff 1986; Michalet et al. 2001; Ober et al. 2004; Snyder et al. 2004;

[Au9] Thompson et al. 2002; Yildiz et al. 2003). The exact expression of the localization accuracy depends on the theoretical framework used to describe the experimental

[Au10] situation. We give here the formula derived in Michalet et al. (2001): 


$$
\sigma=\frac{2}{\sqrt{\pi}} \frac{\delta \mathrm{x}}{\mathrm{SNR}}
$$

where $\delta \mathrm{x}$ is the image pixel size.

Dahan et al. (2003) have shown that it possible to observe QD's SNR of about $\sim 50$ under physiological conditions resulting in accuracy as high as $5-10 \mathrm{~nm}$.

Similarly, Figure 3.5 illustrates how QD's high SNR, brightness, and resistance to photobleaching allow tracking the diffusion of individual QD-tagged CD14 receptors in the membrane of live human HeLa cells (Michalet et al. 2005).

This high-localization accuracy principle together with the broad excitation spectrum of QDs (single excitation, multiple emission, Figure 3.2B) allow us to measure the relative position of two spectrally distinct QDs with nanometer

[Au11] accuracy as well (Lacoste et al. 2000; Michalet et al. 2001). Figure 3.6 presents an example of closely spaced QDs, where centroid-fitting algorithms allow subpixel localization with nanometer accuracy of multiple-color individual QDs (Lacoste et al. 2000). In conclusion, QDs can improve positioning accuracy and distance measurement beyond the diffraction limit imposed by the optical resolution of the microscope, although without increasing the imaging resolution per se.

Improving the optical imaging resolution in $x, y$, and $z$ requires "breaking" the diffraction limit of light. This can only be done by effectively reducing the volume of the PSF. Several methods have been proposed and demonstrated: (i) near-field scanning optical microscopy (NSOM; Betzig and Chichester (1993), Betzig and Trautman (1992); Enderle et al. (1997)) or interference methods such as (ii) standing-wave microscopy (Bailey et al. 1993; Frohn et al. 2000), (iii) I5M (Gustafsson 2005; Gustafsson et al. 1999), (iv) 4Pi microscopy (Egner and Hell 2005; Hell 2003; Hell and Stelzer 1992), and (v) stimulated emission (STED) microscopy (Dyba and Hell 2002; Hofmann et al. 2005; Klar et al. 2000) although the applicability of some of these techniques remain to be demonstrated with QDs.

\subsubsection{Singleness and Blinking}

Because the diffraction-limited volume (typically, several hundred nanometers across, $\sim 1 \mathrm{fl}$ in volume) is much larger than the dimensions of single molecule emitters (dyes and QDs), one has to rule out the possibility that the observed signal originates from two or more emitters. Working at low concentration reduces the probability of finding more than one molecule in the excitation volume. Also, we have seen that the excitation volume could be further reduced by super-resolution methods. Nonetheless, one can only conclude that the observed signal emanates from a single molecule (QD) if the following criteria are met (Betzig and Chichester 1993; Michalet et al. 2003):

The observed (surface or volume) density of emitters correlates with the known concentration of molecules used to prepare the sample.

The observed fluorescence intensity level is consistent with that of a single emitting molecule (according to (Equation 3.2)). 

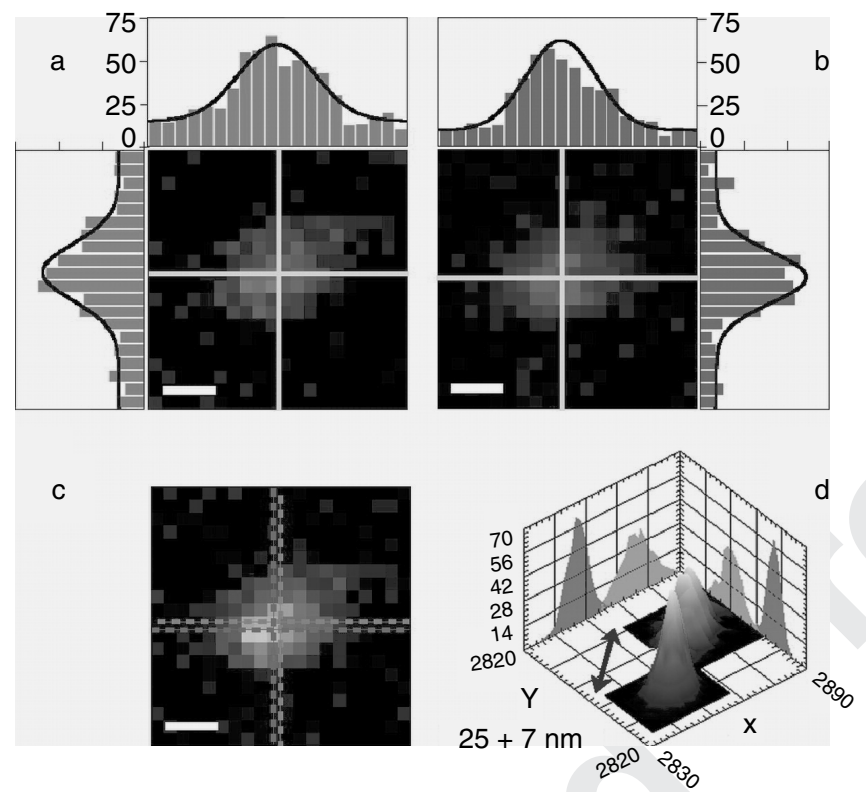

Figure 3.6 Ultrahigh-resolution colocalization of individual nanocrystals. Mixture of green (Em: $540 \mathrm{~nm}$ ) and red (Em: $620 \mathrm{~nm}$ ) QDs excited at $488 \mathrm{~nm}$ (excitation power: $200 \mathrm{nW}$ incident or $320 \mathrm{~W} / \mathrm{cm}^{2}$ peak irradiance; integration time: $50 \mathrm{~ms}$ ). (a), (b) Green and red channel images of a $1 \times 1 \mu \mathrm{m}^{2}$ scan obtained by raster scanning the sample through the fixed excitation PSF and recording the respective signals on two different APDs (pixel size: $50 \mathrm{~nm}$; scale bar: $200 \mathrm{~nm}$ ). As visible from the intensity profiles along two orthogonal lines passing through the PSFs centers, the count rates are similar in both channels. Black curves indicate the corresponding cross-sections of the fitted PSFs. (c) Overlay of the two channels with indication of the determined PSFs centers. (d) Bootstrap replicas of the datasets were fitted in order to estimate the uncertainty of the position determination (Efron and Tibshirani 1994). The figure shows the histograms of the fitted centers distribution obtained from 1000 simulations. The measured distance is $25 \mathrm{~nm}$ with a corresponding uncertainty of $7 \mathrm{~nm}$ (68\% confidence limit). (Reprinted with permission from Lacoste et al. (2000). Copyright 2000, the National Academy of Sciences USA)

When immobilized, each dye or nanocrystal rod emitter has a well-defined absorption or emission dipole (Hu et al. 2001).

Fluorescence emission exhibits only two levels: on/off behavior due to blinking, intersystem crossing to triplet state or photobleaching.

If two or more emission levels exist, they must correlate to changes in photophysical properties (Neuhauser et al. 2000).

The emitted light exhibits antibuching, that is, no simultaneous emission of two photons (Basché et al. 1992). For QDs this is true for low excitation levels only since multiple exciton generation has been recently reported (Ellingson et al. 2005).

Lastly, single QDs can be identified by their characteristic blinking (intermittency) behavior that occurs at all time scales (Banin et al. 1999; Nirmal et al. 1996). 
Intermittency is readily seen by the naked eye using a standard fluorescent microscope. The diffraction-limited spots are seen as alternating between "on" and "off" emission states. Figure 3.5D shows a typical single QD blinking time trace. QD's intermittency has been used as the main criterion for the detection of single QDs in live cells (Chan and Nie 1998; Dahan et al. 2003; Howarth et al. 2005) and fluorescence in situ hybridization (FISH) experiments in fixed cells (Bentolila and Weiss 2006, Crut et al. 2005).

\subsection{Quantum Dots: Applications in Single-Molecule Optical Imaging}

The main benefit of utilizing QDs for single molecule imaging is their enhanced brightness and photostability, that is, the ability to image and observe individual molecules for long periods of time. Up to now this has been exploited to study the dynamics of two classes of molecules: the translocation and conformational changes of mechanochemical transducers (molecular motors), and the diffusion and transport of receptor proteins in the plasma membrane. In the following, we review these recent achievements and speculate on future advances.

\subsubsection{Quantum Dot Imaging of Protein Conformational Changes}

Myosin V is a double-headed molecular motor that delivers intracellular cargo over long distances by moving processively along actin filament tracks. Single-molecule fluorescence polarization (Forkey et al. 2003) and high-accuracy fluorophores localization methods (FIONA; Yildiz et al. (2003)) have suggested that myosin V is likely to walk with a hand-over-hand mechanism where the heads alternate between "leading" and "trailing" positions. Although very convincing, these experiments have, however, relied on monitoring the movement of a single-labeled myosin head with no direct visualization of the movements of the two heads.

Warshaw et al. (2005) used TIR microscopy and the ability to differentially label each head of a single myosin molecule with a QD of a different color to characterize how myosin generates motion under load. The high spatial and temporal resolution afforded in QD imaging allowed the first time observation of each head alternating positions in a hand-over-hand fashion during processive movement. Single-laser excitation and simultaneous detection of the two QD emission spectra allowed precise positioning measurements of the myosin heads in the $x, y$ focal plan with $6 \mathrm{~nm}$ resolution at the $83 \mathrm{~ms}$ integration time (Figure 3.7). These data, through direct observation, confirmed myosin V's hand-over-hand mode of walking along an actin filament. The authors demonstrated that the QD load did not affect the overall velocity of the motor. They also observed hidden substeps suggesting that 


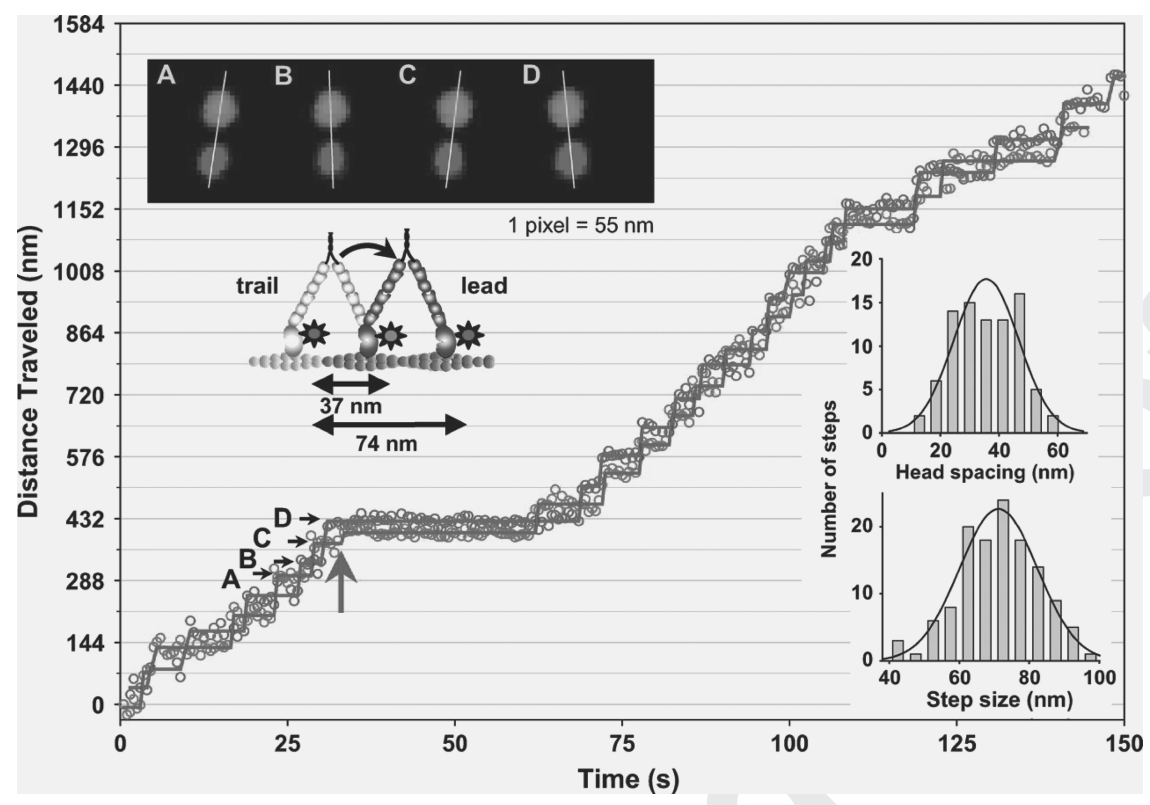

Figure 3.7 Myosin V processive run with heads labeled with different colored quantum dots. Green and red open circles are the $\mathrm{QD}_{565}$ and $\mathrm{QD}_{655}$ positions, respectively, determined by Gaussian fits. Solid lines are the average QD positions between steps with the onset of steps determined by eye. Upper left are averaged QD images for steps labeled $A-D$, with red and green images offset by 12 pixels in $y$ for clarity. The yellow lines connect QD centers emphasizing alternating relative head positions. Green arrow identifies substep. Lower right are histograms of interhead spacing and step size. (Reprinted with permission from (Warshaw et al. 2005). Copyright 2005 American Biophysical Society)

once the head has stably attached to an actin monomer, it can detach and reattach before taking its following step.

Another important issue is to understand how motor proteins carry out their functions in living cells under physiological conditions. Beside a single report using a green fluorescence protein (GFP), high background noise, rapid photobleaching, and poor spatial resolution have so far precluded resolving the individual steps of molecular motor proteins in vivo. To circumvent these limitations, Nan et al. (2005) have taken advantage of the exceptional brightness and photostability of QDs and recently reported the first observation of individual microtubule motor steps in living cells by following the movements of endocytic vesicles loaded with QDs. The authors showed that QDs allowed them to record motor displacement traces with $300 \mu$ s time resolution and $1.5 \mathrm{~nm}$ spatial precision. They also documented new aspects of microtubule motor movements that had been previously undetected using conventional dye technology.

These results demonstrate the ability of QDs to monitor motor proteins at the molecular level in living cells with higher resolution and will certainly be followed by studies on other motor proteins and systems. 


\subsubsection{Quantum Dot Tracking of Cell-Surface Membrane Receptors}

Monitoring the trajectories of individual proteins in the plasma membrane of cells has been limited so far by either the size of the probe $(40 \mathrm{~nm}$ gold nanoparticles or $500 \mathrm{~nm}$ latex beads) that may interfere with protein dynamic (Saxton and Jacobson 1997 ) or by the rapid photobleaching of small (1 to $4 \mathrm{~nm}$ ) fluorescent organic labels (Ueda et al. 2001).

To circumvent these two main limitations, Dahan et al. (2003) have used antibodyconjugated QDs to track individual glycine receptors at the surface of living neurons. The authors used a standard wide-field microscope and a sensitive CCD camera to identify individual QD-tagged membrane receptors according to their blinking properties. The distance separating neighboring QDs (i.e., the density of membrane receptor labeling) was carefully optimized to make sure that individual QDs could be distinguished optically (i.e., less than one labeled receptor per diffraction-limited resolved spot).

Although the trajectory of an individual receptor could not necessarily be tracked continuously due to blinking (Figure 3.8), the advantages of using QDs were evident. QDs' photostability allowed the observation of individual receptor trajectory for at least $20 \mathrm{~min}$ compared to $\sim 5 \mathrm{~s}$ with Cy3-labeled antibodies. The brightness of the QDs allowed the spots to be detected with a SNR of about 50 (integration time $75 \mathrm{~ms}$ ), an increase of almost a factor of 10. This directly translated into a better positioning accuracy of $\sim 5-10 \mathrm{~nm}$ (see Equation (3.8)). These experiments also revealed that extrasynaptic receptors were far more mobile than previously considered by single-particle tracking using micrometer-sized beads (Meier et al. 2001), further confirming that the larger load size does influence diffusion properties. Finally, the authors demonstrated the ability to combine the high-temporal dynamics afforded by single-molecule fluorescence microscopy

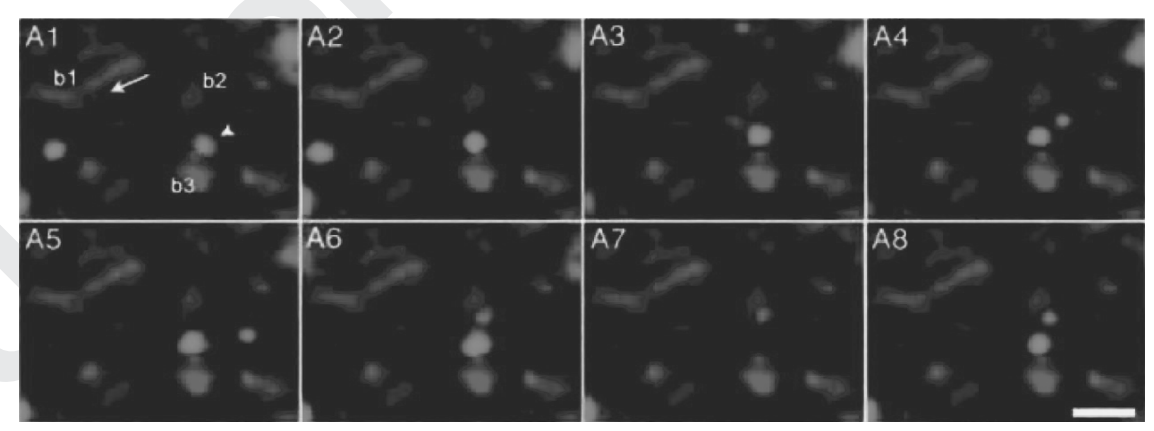

Figure 3.8 Motion of individual glycine receptors diffusing in the membrane of live neurons. Red spots correspond to synaptic boutons labeled with FM 4-64. One QD (arrow), initially located in bouton b1 diffuses in the extrasynaptic domain and then associates with bouton b2. A second QD (arrowhead) diffuses while remaining associated with bouton b3. Scale bar is $2 \mu \mathrm{m}$. (Reprinted with permission from Dahan et al. (2003). Copyright 2003 AAAS) 
with high-resolution cellular localization afforded by electron microscopy on the same QD-labeled samples.

\subsection{Future Directions}

The two previous examples clearly illustrate the unmatched potential of QD technology for single-molecule microscopy and spectroscopy. It is reasonable to expect that with further experimental optimization (microscope geometries, QD photophysical properties, and/or development of new detectors) high positioning accuracy measurement could be approaching or surpass the one-and-half nanometer precision that has been achieved with single-color organic dyes (Snyder et al. 2004, Yildiz et al. 2003). Furthermore, the demonstration that a polarization microscope allows the detection of the QDs and nanorods orientation in three-dimensional

[Au12] space (Empedocles et al. 1999; Hu, 2001, p.131) suggests new methods to study protein undergoing conformational changes in real-time by enlisting the use of endspecific functionalized nanorods (Mokari et al. 2004).

QDs may also be instrumental in achieving single-molecule visualization of the motion of intracellular proteins involved in signaling pathways. Indeed, the observation of single molecules inside living cells has remained challenging partially due to the cellular autofluorescence originating from natural cellular fluorophores present in organelles (flavins, NADH, etc.). This inherent limitation might be alleviated by imaging long-wavelength QDs (extending to the NIR spectrum where autofluorescence is reduced) and/or by taking advantage of the long QD fluorescence lifetime ( $>5 \mathrm{~ns}$; Efros and Rosen (2000)) to combine the power of time-gated detection (Dahan et al. 2001) to further separate the QD fluorescent signal from that of the cellular background fluorescence (constituted mostly of shorter-lived species).

A prerequisite for QD intracellular applications is the determination of reproducible conditions to deliver monodisperse QDs inside the cell cytoplasm that avoid aggregation in the endosomes (Chen and Gerion 2004; Derfus et al. 2004). It might be also necessary to associate QDs with a fluorescence-quenching molecule that can dissociate upon binding to the target (Chang et al. 2005) to allow discriminating between bound and unbound QDs. Finally, a new software/hardware combination might be needed to track the motion of intracellular molecules that is likely to take place within the three-dimensional space of the cytosol (Levi et al. 2005; Speidel et al. 2003).

Although not achieving single-molecule detection yet, Lidke et al. (2004, 2005) have provided "proof-of-concept" studies showing that QDs are indeed powerful tools to continuously monitor complex signaling pathways, from the cell membrane to specific organelles in the cytoplasm. Lidke et al. used QD-tagged epidermal growth factor (EGF) to directly image the ligand-induced activation process as well as the homo- and heterodimerization of members of the erbB receptor family. QD's photostability over more than 60 min allowed continuous tracking of ErbB1 receptors from the cell membrane, through endocytosis and the subsequent pathways of vesicular trafficking and fusion. 
It is important to note that when single-molecule sensitivity could be achieved at the surface of the filipodia, the authors discovered a new retrograde transport of the erbB1 receptor associated with the cytoskeleton. This retrograde transport preceded receptor endocytosis, which occurs at the base of the filipodia (Lidke et al. 2005).

\subsection{Concluding Remarks}

Since their introduction in 1998 (Bruchez et al. 1998; Chan and Nie 1998), fluorescent semiconductor nanocrystals have been widely accepted by the scientific community and the biotechnology industry as new fluorescent, nonisotopic labels of unmatched potential. Although QDs are here to stay, they are not likely to replace fluorescent dyes and genetically encoded proteins technologies. Rather, they will complement some of their shortcomings in niche applications requiring better photostability, NIR emission, multiplexing (multicolor) capabilities, and single-molecule sensitivity over long periods of time. Quite remarkably, quantum confinement phenomena in nanoscale materials, first predicted and studied by physicists, next synthesized and implemented by inorganic chemists, are now being harnessed and utilized by biologists as a new tool to visualize and study functional molecules down to the single-molecule level within cells and organisms.

Acknowledgments The development of QDs and SM microscopy were made possible thanks to the contribution of past and present members of our laboratory and our valuable colleagues in the field. We would also like to thank Tal Paley for her editorial assistance. Fluorescent microscopy images in Figure 3.5 were obtained at the California NanoSystems Institute Advanced Light Microscopy/Spectroscopy Shared Facility, Department of Chemistry and Biochemistry, at UCLA. This work was supported by funds from the National Institute of Health, Grant No. 5-R01-EB000312-04 and by the Department of Energy, Grant No. ER63421-0008273.

\section{References}

Abbe E (1873) Beitrage zur Theorie des Mikroskops und der mikroskopischen Wahrnehmung. Arch. f. Mikroskop. Anat. 9: 413-420

Akerman ME, Chan WCW, Laakkonen P, Bhatia SN, and Ruoslahti E (2002) Nanocrystal targeting in vivo. Proc. Natl. Acad. Sci. USA 99 (20): 12617-12621

Alivisatos AP (1996a) Semiconductor clusters, nanocrystals, and quantum dots. Science 271 (5251): 933-937

Alivisatos AP (1996b) Perspectives on the physical chemistry of semiconductor nanocrystals. $J$. Phys. Chem.100 (31): 13226-13239

Alivisatos P (2004) The use of nanocrystals in biological detection. Nat. Biotechnol. 22 (1): $47-52$

Ambrose WP and Moerner WE (1991) Fluorescence spectroscopy and spectral diffusion of single impurity molecules in a crystal. Nature 349 : 225-227

Ashoori RC (1996) Electrons in artificial atoms. Nature 379: 413-419 
Atkins PW and Friedman RS (1997) Molecular Quantum Mechanics. Oxford University Press, New York

Axelrod D (2003) Total internal reflection fluorescence microscopy in cell biology. Meth. Enzymol. 361: 1-33

Bailey B, Farkas DL, Taylor DL, and Lanni F (1993) Enhancement of axial resolution in fluorescence microscopy by standing-wave excitation. Nature 366 (6450): 44-48

Ballou B, Lagerholm BC, Ernst LA, Bruchez MP, and Waggoner AS (2004) Noninvasive imaging of quantum dots in mice. Bioconjug. Chem. 15 (1): 79-86

Banin U, Bruchez M, Alivisatos AP, Ha T, Weiss S, and Chemla DS (1999) Evidence for a thermal contribution to emission intermittency in single $\mathrm{CdSe} / \mathrm{CdS}$ core/shell nanocrystals. J. Chem. Phys. 110 (2): 1195-1201

Basché T, Moerner WE, Orrit M, and Talon H (1992) Photon antibunching in the fluorescence of a single dye molecule trapped in a solid. Phys. Rev. Lett. 69: 1516-1519

Bentolila LA and Weiss S (2006) Single-step multicolor fluorescent in situ hybridization analysis using semiconductor quantum dot-DNA conjugates. Cell Biochem. Biophys. 44 (1): in press

Betzig E and Chichester RJ (1993) Single molecules observed by near-field scanning optical microscopy. Science 262: 1422-1425

Betzig E and Trautman JK (1992) Near-field optics: Microscopy, spectroscopy, and surface modification beyond the diffraction limit. Science 257: 189-195

Bobroff N (1986) Position measurement with a noise-limited instrument. Rev. Sci. Instrum. 57 (6): $1152-1157$

Böer KW (1990) Survey of Semiconductor Physics. Electrons and Other Particles in Bulk Semiconductors. Van Nostrand Reinhold, New York

Bowen Katari JEB, Colvin VL, and Alivisatos AP (1994) X-ray photoelectron spectroscopy of CdSe nanocrystals with applications to studies of the nanocrystal surface. J. Phys. Chem. 98: 4109-4117

Bruchez M, Moronne M, Gin P, Weiss S and Alivisatos AP (1998) Semiconductor nanocrystals as fluorescent biological labels. Science 281: 2013-2015

Brus LE (1984) Electron-electron and electron-hole interactions in small semiconductor crystallites: the size dependence of the lowest excited electronic state. J. Chem. Phys. 80 (9): 4403-9

Chan P, Yuen T, Ruf F, Gonzalez-Maeso J, and Sealfon SC (2005) Method for multiplex cellular detection of mRNAs using quantum dot fluorescent in situ hybridization. Nucleic Acids Res. 33 (18): e161

Chan WCW and Nie SM (1998) Quantum dot bioconjugates for ultrasensitive nonisotopic detection. Science 281 (5385): 2016-2018

Chang E, Miller JS, Sun J, Yu WW, Colvin VL, Drezek R, and West JL (2005) Protease-activated quantum dot probes. Biochem. Biophys. Res. Commun. 334 (4): 1317-1321.

Chen F and Gerion D (2004) Fluorescent CdSe/ZnS nanocrystal-peptide conjugates for long-term, nontoxic imaging and nuclear targeting in living cells. Nano Lett. 4: 1827-1832

Cobbett CS (2001) Heavy metal detoxification in plants: Phytochelatin biosynthesis and function. Iubmb Life 51 (3): 183-188

Crut A, Geron-Landre B, Bonnet I, Bonneau S, Desbiolles P, and Escude C (2005) Detection of single DNA molecules by multicolor quantum-dot end-labeling. Nucleic Acids Res. 33 (11): e98

Dabbousi RO, Rodriguez-Viejo J, Mikulec FV, Heine JR, Mattoussi H, Ober R, Jensen KF, and Bawendi MG (1997) (CdSe)ZnS core-shell quantum dots: Synthesis and characterization of a size series of highly luminescent nanocrystallites. J. Phys. Chem. B 101: 9463-9475

Dahan M, Laurence T, Pinaud F, Chemla DS, Alivisatos AP, Sauer M, and Weiss S (2001) Timegated biological imaging by use of colloidal quantum dots. Optics Lett. 26 (11): 825-827

Dahan M, Levi S, Luccardini C, Rostaing P, Riveau B, and Triller A (2003) Diffusion dynamics of glycine receptors revealed by single-quantum dot tracking. Science 302 (5644): 442-445

Dameron CT and Winge DR (1990a) Characterization of peptide-coated cadmium-sulfide crystallites. Inorg. Chem. 29 (7): 1343-1348 
Dameron CT and Winge DR (1990b) Peptide-mediated formation of quantum semiconductors. Trends Biotechnol. 8 (1): 3-6

Dameron CT, Reese RN, Mehra RK, Kortan AR, J. CP, Steigerwald ML, Brus LE, and Winge DR (1989) Biosynthesis of cadmium sulphide quantum semiconductor crystallites. Nature 338: 596-597

Derfus AM, Chan WCW, and Bhatia SN (2004) Intracellular delivery of quantum dots for live cell labeling and organelle tracking. Adv. Mater. 16 (12): 961-966

Doose S, Tsay JM, Pinaud F, and Weiss S (2005) Comparison of photophysical and colloidal properties of biocompatible semiconductor nanocrystals using fluorescence correlation spectroscopy. Anal. Chem. 77 (7): 2235-2242

Dubertret B, Skourides P, Norris DJ, Noireaux V, Brivanlou AH, and Libchaber A (2002) In vivo imaging of quantum dots encapsulated in phospholipid micelles. Science 298 (5599): $1759-1762$

Dyba M and Hell SW (2002) Focal spots of size lambda/23 open up far-field fluorescence microscopy at $33 \mathrm{~nm}$ axial resolution. Phys. Rev. Lett. 88 (16): 163901

Efron B and Tibshirani RJ (1994) An Introduction to the Bootstrap. CRC Press, Boca Raton, FL.

Efros AL and Efros AL (1982) Interband absorption of light in a semiconductor sphere. Sov. Phys. Semicond. 16 (7): 1209-1214

Efros AL and Rosen M (2000) The electronic structure of semiconductor nanocrystals. Ann. Rev. Mat. Sci. 30: 475-521

Egner A and Hell SW (2005) Fluorescence microscopy with super-resolved optical sections. Trends Cell Biol. 15 (4): 207-215

Ekimov AI and Onuschenko AA (1982) Quantum size effect in the optical spectra of semiconductor microcrystals. Sov. Phys. Semicond. 16 (7): 1215-1219

Ekimov AI, Onuschenko AA, and Tsekhomskii VA (1980) Exciton light absorption by $\mathrm{CuCl}$ microcrystals in glass matrix. Sov. Glass Phys. Chem. 6: 511-512

Ellingson RJ, Beard MC, Johnson JC, Yu P, Micic OI, Nozik AJ, Shabaev A, and Efros AL (2005) Highly efficient multiple exciton generation in colloidal $\mathrm{PbSe}$ and $\mathrm{PbS}$ quantum dots. Nano Lett. 5 (5): 865-871

Empedocles S and Bawendi M (1999) Spectroscopy of single CdSe nanocrystallites. Accounts Chem. Res. 32 (5): 389-396

Empedocles SA, Neuhauser R, Shimizu K, and Bawendi MG (1999) Photoluminescence from single semiconductor nanostructures. Adv. Mater. 11: 1243-1256

Enderle T, Ha T, Ogletree DF, Chemla DS, Magowan C, and Weiss S (1997) Membrane specific mapping and colocalization of malarial and host skeletal proteins in the Plasmodium falciparum infected erythrocyte by dual-color near-field scanning optical microscopy. Proc Natl Acad Sci U S A 94 (2): 520-525

Eychmüller A, Mews A, and Weller H (1993) A quantum dot quantum well: CdS/HgS/CdS. Chem. Phys. Lett. 208: 59-62

Forkey JN, Quinlan ME, Shaw MA, Corrie JE, and Goldman YE (2003) Three-dimensional structural dynamics of myosin V by single-molecule fluorescence polarization. Nature 422 (6930): 399-404

Frohn JT, Knapp HF, and Stemmer A (2000) True optical resolution beyond the Rayleigh limit achieved by standing wave illumination. Proc Natl Acad Sci U S A 97 (13): 7232-7236

Funatsu T, Harada Y, Tokunaga M, Saito K, and Yanagida T (1995) Imaging of single fluorescent molecules and individual ATP turnovers by single myosin molecules in aqueous solution. Nature 374 (6522): 555-559

Gao X, Chan WCW, and Nie S (2002) Quantum-dot nanocrystals for ultrasensitive biological labeling and multicolor optical encoding. J. Biomed. Opt. 7 (4): 532-537

Gao X, Cui Y, Levenson RM, Chung LWK, and Nie S (2004) In vivo cancer targeting and imaging with semiconductor quantum dots. Nature Biotechnol. 22: 969

Gaponenko SV (1998) Optical Properties of Semiconductor Nanocrystals. Cambridge University Press, Cambridge 
Gaponik N, Talapin DV, Rogach AL, Hoppe K, Shevchenko EV, Kornowski A, Eychmüller A, and Weller H (2002) Thiol-capping of CdTe nanocrystals: an alternative to organometallic synthetic routes. J. Phys. Chem. B 106: 7177-7185

Gelles J, Schnapp BJ, and Sheetz MP (1988) Tracking kinesin-driven movements with nanometer scale precision. Nature 331: 450-453

Gerion D, Pinaud F, Williams SC, Parak WJ, Zanchet D, Weiss S, and Alivisatos AP (2001) Synthesis and properties of biocompatible water-soluble silica-coated $\mathrm{CdSe} / \mathrm{ZnS}$ semiconductor quantum dots. J. Phys. Chem. B 105 (37): 8861-8871

Goldman ER, Anderson GP, Tran PT, Mattoussi H, Charles PT, and Mauro JM (2002) Conjugation of luminescent quantum dots with antibodies using an engineered adaptor protein to provide new reagents for fluoroimmunoassays. Anal. Chem. 74 (4): 841-847

Goulian M and Simon SM (2000) Tracking single proteins within cells. Biophys. J. 79 $2188-2198$

Guo W, Li JJ, Wang YA, and Peng XG (2003) Conjugation chemistry and bioapplications of semiconductor box nanocrystals prepared via dendrimer bridging. Chem. Mater. 15 (16): 3125-3133

Gustafsson MG (2005) Nonlinear structured-illumination microscopy: wide-field fluorescence imaging with theoretically unlimited resolution. Proc. Natl. Acad. Sci. USA 102 (37): 13081-6

Gustafsson MG, Agard DA, and Sedat JW (1999) I5M: 3D widefield light microscopy with better than $100 \mathrm{~nm}$ axial resolution. J. Microsc. 195 (Pt 1): 10-16

Hannay NB (1959) Semiconductor. Reinhold, New York

Harms GS, Cognet L, Lommerse PH, Blab GA, Kahr H, Gamsjager R, Spaink HP, Soldatov NM, Romanin C, and Schmidt T (2001) Single-molecule imaging of l-type $\mathrm{Ca}(2+)$ channels in live cells. Biophys J. 81 (5): 2639-2646

Hell SW (2003) Toward fluorescence nanoscopy. Nature Biotechnol. 21 (11): 1347-1355

Hell SW and Stelzer EHK (1992) Properties of a 4Pi confocal fluorescence microscope. J. Optic. Soc. Amer. 9: 2159-2166

Hines MA and Guyot-Sionnest P (1996) Synthesis and characterization of strongly luminescing ZnS-capped CdSe nanocrystals. J. Phys. Chem. 100 468-471

Hirschfeld T (1976) Optical microscopic observation of single small molecules. Appl. Optics 15 (12): 2965-2966

Hofmann M, Eggeling C, Jakobs S, and Hell SW (2005) Breaking the diffraction barrier in fluorescence microscopy at low light intensities by using reversibly photoswitchable proteins. Proc. Natl. Acad. Sci. USA 102 (49): 17565-17569

Hohng S and Ha T (2004) Near-complete suppression of quantum dot blinking in ambient conditions. J. Am. Chem. Soc. 126 (5): 1324-5

Howarth M, Takao K, Hayashi Y and Ting AY (2005) Targeting quantum dots to surface proteins in living cells with biotin ligase. Proc. Natl. Acad. Sci. USA 102 (21): 7583-7588

Hu J, Li L, Yang W, Manna L, Wang L, and Alivisatos AP (2001) Linearly polarized emission from colloidal semiconductor quantum rods. Science 292 (5524): 2060-3

Iino R, Koyama I and Kusumi A (2001) Single molecule imaging of green fluorescent proteins in living cells: E-cadherin forms oligomers on the free cell surface. Biophys. J. 80 (6): 2667-2677

Jaiswal JK, Mattoussi H, Mauro JM, and Simon SM (2003) Long-term multiple color imaging of live cells using quantum dot bioconjugates. Nature Biotechnol. 21 (1): 47-51

Kadavanich A, V., Kippeny TC, Erwin MM, Pennycook SJ and Rosenthal SJ (2001) Sublattice resolution structural and chemical analysis of individual CdSe nanocrystals using atomic number contrast scanning transmission electron microscopy and electron energy loss spectroscopy. J. Phys. Chem. B 105 361-369

Kapanidis A and Weiss S (2002) Fluorescent probes and bioconjugation chemsitries for singlemolecule fluorescence analysis of biomolecules. J. Chem. Phys. 117 10953-10964

Kellermayer MS (2005) Visualizing and manipulating individual protein molecules. Physiol. Meas. 26 (4): R119-153 
Kim S and Bawendi MG (2003) Oligomeric ligands for luminescent and stable nanocrystal quantum dots. J. Am. Chem. Soc. 125 (48): 14652-14653

Kim S, Lim YT, Soltesz EG, De Grand AM, Lee J, Nakayama A, Parker JA, Mihaljevic T, Laurence RG, Dor DM, Cohn LH, Bawendi MG, and Frangioni JV (2004) Near-infrared fluorescent type II quantum dots for sentinel lymph node mapping. Nature Biotechnol. 22 (1): 93-97

Kittel C (1996) Introduction to Solid State Physics. John Wiley, New York

Klar TA, Jakobs S, Dyba M, Egner A, and Hell SW (2000) Fluorescence microscopy with diffraction resolution barrier broken by stimulated emission. Proc. Nat.Acad. Sci. USA 97: 8206-8210

Kortan AR, Hull R, Opila RL, Bawendi MG, Steigerwald ML, Carroll PJ, and Brus LE (1990) Nucleation and growth of CdSe on $\mathrm{ZnS}$ quantum crystallite seeds, and vice versa, in inverse micelle media. J. Amer. Chem. Soc. 112: 1327-1332

Lacoste TD, Michalet X, Pinaud F, Chemla DS, Alivisatos AP, and Weiss S (2000) Ultrahighresolution multicolor colocalization of single fluorescent probes. Proc. Natl. Acad. Sci. USA 97 (17): 9461-9466

Larson D, Zipfel W, Williams R, Clark S, Bruchez M, Wise F, and Webb WW (2003) Water-soluble quantum dots for multiphoton fluorescence imaging in vivo. Science 300: 1434-1436

Leatherdale CA, Woo WK, Mikulec FV, and Bawendi MG (2002) On the absorption cross section of CdSe nanocrystal quantum dots. J. Phys. Chem. B 106: 7619-7622

Levi V, Ruan Q, and Gratton E (2005) 3-D particle tracking in a two-photon microscope: Application to the study of molecular dynamics in cells. Biophys. J. 88 (4): 2919-2928

Li JJ, Tsay JM, Michalet X, and Weiss S (2005) Wavefunction engineering: From quantum wells to near-infrared type-II colloidal quantum dots synthesized by layer-by-layer colloidal epitaxy. Chem. Phys. 318 (1-2): 82-90

Lidke DS, Lidke KA, Rieger B, Jovin TM, and Arndt-Jovin DJ (2005) Reaching out for signals: Filopodia sense EGF and respond by directed retrograde transport of activated receptors. $J$. Cell Biol. 170 (4): 619-26

Lidke DS, Nagy P, Heintzmann R, Arndt-Jovin DJ, Post JN, Grecco HE, Jares-Erijman EA, and Jovin TM (2004) Quantum dot ligands provide new insights into erbB/HER receptor-mediated signal transduction. Nat. Biotechnol. 22 (2): 198-203

Macklin JJ, Trautman JK, Harris TD, and Brus LE (1996) Imaging and time-resolved spectroscopy of single molecules at an interface. Science 272: 255-258

Mahan GD (2000) Many-Particle Physics. Kluwer Academic/Plenum, New York

Mansson A, Sundberg M, Balaz M, Bunk R, Nicholls IA, Omling P, Tagerud S, and Montelius L (2004) In vitro sliding of actin filaments labelled with single quantum dots. Biochem. Biophys. Res. Commun. 314 (2): 529-534

Mattoussi H, Mauro JM, Goldman ER, Anderson GP, Sundar VC, Mikulec FV, and Bawendi MG (2000) Self-assembly of CdSe-ZnS quantum dot bioconjugates using an engineered recombinant protein. J. Am. Chem. Soc. 122 (49): 12142-12150

Medintz IL, Uyeda HT, Goldman ER, and Mattoussi H (2005) Quantum dot bioconjugates for imaging, labelling and sensing. Nat. Mater. 4 (6): 435-46

Meier J, Vannier C, Serge A, Triller A, and Choquet D (2001) Fast and reversible trapping of surface glycine receptors by gephyrin. Nat. Neurosci. 4 (3): 253-60

Mews A, Eychmüller A, Giersig M, Schoos D, and Weller H (1994) Preparation, characterization, and photophysics of the quantum dot quantum well system $\mathrm{CdS} / \mathrm{HgS} / \mathrm{CdS}$. J. Phys. Chem. 98: 934-941

Michalet X, Kapanidis AN, Laurence T, Pinaud F, Doose S, Pflughoefft M, and Weiss S (2003) The power and prospects of fluorescence microscopies and spectroscopies. Ann. Rev. Biophys. Biomol. Struct. 32: 161-182

Michalet X, Lacoste TD, and Weiss S (2001a) Ultrahigh-resolution colocalization of spectrally resolvable point-like fluorescent probes. Methods 25 (1):

Michalet X, Pinaud F, Lacoste TD, Dahan M, Bruchez MP, Alivisatos AP, and Weiss S (2001b) Properties of fluorescent semiconductor nanocrystals and their application to biological labeling. Single Mol. 2 (4): 261-276 
Michalet X, Pinaud FF, Bentolila LA, Tsay JM, Doose S, Li JJ, Sundaresan G, Wu AM, Gambhir SS, and Weiss S (2005) Quantum dots for live cells, in vivo imaging, and diagnostics. Science 307 (5709): 538-544

Mitchell GP, Mirkin CA, and Letsinger RL (1999) Programmed assembly of DNA functionalized quantum dots. J. Am. Chem. Soc. 121 (35): 8122-8123

Moerner WE (2003) Optical measurements of single molecules in cells. Trends Anal. Chem. 22 (9): 544-548

Mokari T, Rothenberg E, Popov I, Costi R, and Banin U (2004) Selective growth of metal tips onto semiconductor quantum rods and tetrapods. Science 304 (5678): 1787-1790

Murphy CJaC, J.L. (2002) Quantum dots: A primer. Appl. Spectroscopy 56 (1): 16A-27A

Murray CB, Norris DJ, and Bawendi MG (1993) Synthesis and characterization of nearly monodisperse $\mathrm{CdE}(\mathrm{E}=\mathrm{S}, \mathrm{Se}, \mathrm{Te})$ semiconductor nanocrystallites. J. Amer. Chem. Soc. 115: 8706-8715

Nan X, Sims PA, Chen P, and Xie XS (2005) Observation of individual microtubule motor steps in living cells with endocytosed quantum dots. J. Phys. Chem. B Condens. Matter Mater. Surf. Interfaces Biophys. 109 (51): 24220-24224

Neuhauser RG, Shimizu KT, Woo WK, Empedocles SA, and Bawendi MG (2000) Correlation between fluorescence intermittency and spectral diffusion in single semiconductor quantum dots. Phys. Rev. Lett. 85 (15): 3301-3304

Nirmal M, Dabbousi BO, Bawendi MG, Macklin JJ, Trautman JK, Harris TD, and Brus LE (1996) Fluorescence intermittency in single cadmium selenide nanocrystals. Nature 383: 802-804

Ober RJ, Ram S, and Ward ES (2004) Localization accuracy in single-molecule microscopy. Biophys. J. 86 (2): 1185-1200

Orrit M and Bernard J (1990) Single pentacene molecules detected by fluorescence excitation in a p-terphenyl crystal. Phys. Rev. Lett. 65 (21): 2716-2719

Osaki F, Kanamori T, Sando S, Sera T, and Aoyama Y (2004) A quantum dot conjugated sugar ball and its cellular uptake. On the size effects of endocytosis in the subviral region. J. Am. Chem. Soc. 126: 6520-6521

Paige MF, Bjerneld EJ, and Moerner WE (2001) A comparison of through-the-objective total internal reflection microscopy and epifluorescence microscopy for single-molecule fluorescence imaging. Single Molec. 2: 191-201

Pathak S, Choi SK, Arnheim N, and Thompson ME (2001) Hydroxylated quantum dots as luminescent probes for in situ hybridization. J. Am. Chem. Soc. 123 (17): 4103-4104

Pellegrino T, Manna L, Kudera S, Liedl T, Koktysh D, Rogach AL, Keller S, Radler J, Natile G, and Parak WJ (2004) Hydrophobic nanocrystals coated with an amphiphilic polymer shell: A general route to water soluble nanocrystals. Nano Lett. 4 (4): 703-707

Peng X, Schlamp MC, Kadavanich AV, and Alivisatos AP (1997) Epitaxial growth of highly luminescent $\mathrm{CdSe} / \mathrm{CdS}$ core/shell nanocrystals with photostability and electronic accessibility. J. Amer. Chem. Soc. 119 (30): 7019-7029

Pinaud F, King D, Moore H-P, and Weiss S (2004) Bioactivation and cell targeting of semiconductor $\mathrm{CdSe} / \mathrm{ZnS}$ nanocrystals with phytochelatin-related peptides. J. Am. Chem. Soc. 126 6115-6123

Reiss P, Bleuse J, and Pron A (2002) Highly luminescent CdSe/ZnSe core/shell nanocrystals of low size dispersion. Nano Lett. 2 (7): 781-784

Rogach AL, Harrison MT, Kershaw SV, Kornowski A, Burt MG, Eychmuller A, and Weller H (2001) Colloidally prepared $\mathrm{CdHgTe}$ and $\mathrm{HgTe}$ quantum dots with strong near-infrared luminescence. Phys. Status Solidi B 224 (1): 153-158

Rossetti R, Nakahara S, and Brus LE (1983) Quantum size effects in the redox potentials, resonance Raman spectra, and electronic spectra of CdS crystallites in aqueous solution. J. Chem. Phys. 79: 1086-1088

Saxton MJ and Jacobson K (1997) Single-particle tracking: Applications to membrane dynamics. Ann. Rev. Biophys. Biomol. Struct. 26: 373-399

Schmidt T, Schütz GJ, Baumgartner W, Gruber HJ, and Schindler H (1996) Imaging of single molecule diffusion. Proc. Nat. Acad. Sci. USA 93: 2926-2929 
Schutz GJ, Kada G, Pastushenko VP, and Schindler H (2000) Properties of lipid microdomains in a muscle cell membrane visualized by single molecule microscopy. Embo. J. 19 (5): 892-901

Seisenberger G, Ried MU, Endress T, Buning H, Hallek M, and Brauchle C (2001) Real-time single-molecule imaging of the infection pathway of an adeno-associated virus. Science 294 (5548): 1929-32

Snyder GE, Sakamoto T, Hammer JA, 3rd, Sellers JR, and Selvin PR (2004) Nanometer localization of single green fluorescent proteins: Evidence that myosin $\mathrm{V}$ walks hand-over-hand via telemark configuration. Biophys. J. 87 (3): 1776-1783

Speidel M, Jonas A, and Florin EL (2003) Three-dimensional tracking of fluorescent nanoparticles with subnanometer precision by use of off-focus imaging. Opt. Lett. 28 (2): 69-71

Steigerwald ML and Brus LE (1990) Semiconductor crystallites: A class of large molecules. Acc. Chem. Res. 23 (6): 183-8

Stillman MJ (1995) Metallothioneins. Coord. Chem. Rev. 144: 461-511

Sukhanova A, Devy M, Venteo L, Kaplan H, Artemyev M, Oleinikov V, Klinov D, Pluot M, Cohen JHM, and Nabiev I (2004) Biocompatible fluorescent nanocrystals for immunolabeling of membrane proteins and cells. Anal. Biochem. 324 (1): 60-67

Thompson RE, Larson DR, and Webb WW (2002) Precise nanometer localization analysis for individual fluorescent probes. Biophys. J. 82 (5): 2775-83

Ueda M, Sako Y, Tanaka T, Devreotes P, and Yanagida T (2001) Single-molecule analysis of chemotactic signaling in Dictyostelium cells. Science 294 (5543): 864-7

Vossmeyer T, Katsikas L, Giersig M, Popovic IG, Diesner K, Chemseddine A, Eychmuller A, and Weller H (1994) CdS nanoclusters: Synthesis, characterization, size dependent oscillator strength, temperature shift of the excitonic transition energy, and reversible absorbance shift. J. Phys. Chem. 98: 7665-7673

Warshaw DM, Kennedy GG, Work SS, Krementsova EB, Beck S, and Trybus KM (2005) Differential labeling of myosin $\mathrm{V}$ heads with quantum dots allows direct visualization of handover-hand processivity. Biophys. J. 88 (5): L30-32

Webb RH (1999) Theoretical basis of confocal microscopy. Methods Enzymol. 307: 3-20

Weiss S (1999) Fluorescence spectroscopy of single biomolecules. Science 283: 1676-1683

Weller H (1993a) Quantized semiconductor particles: A novel state of matter for materials science. Adv. Mater. (Weinheim, Germany) 5 (2): 88-95

Weller H (1993b) Colloidal semiconductor Q-particles: Chemistry in the transition region between solid and molecular states. Angewandte Chemie 105 (1): 43-55 (See also Angew. Chem., Int. Ed. Engl., 1993, 32(1), 41-53)

Weller H (1998) Quantum size colloids: From size-dependent properties of discrete particles to self-organized superstructures. Curr. Opin. Colloid Interface Sci. 3 (2): 194-199

Wu XY, Liu HJ, Liu JQ, Haley KN, Treadway JA, Larson JP, Ge NF, Peale F, and Bruchez MP (2003) Immunofluorescent labeling of cancer marker Her2 and other cellular targets with semiconductor quantum dots. Nature Biotechnol. 21 (1): 41-46

Yildiz A, Forkey JN, McKinney SA, Ha T, Goldman YE, and Selvin PR (2003) Myosin V walks hand-over-hand: Single fluorophore imaging with 1.5-nm localization. Science 300 (5628): 2061-2065

Yin Y and Alivisatos AP (2005) Colloidal nanocrystal synthesis and the organic-inorganic interface. Nature 437 (7059): 664-70

[Au13] Zhang JZ (1997) Ultrafast studies of electron dynamics in semiconductor and metal colloidal nanoparticles: Effects of size and surface. Acc. Chem. Res. 30 (10): 423-429

\section{Author Queries:}

[Au1]: Please include in Refs

[Au2]: Sentence OK? 
[Au3]: Please indicate a or b [Au4]: Please include in Refs [Au5]: Please indicate a or b [Au6]: Please include in Refs [Au7]: Please include in Refs [Au8]: Please indicate 2001a or b [Au9]: Please indicate 2001a or b [Au10]: Please indicate a or b [Au11]: 2001a or b?

[Au12]: OK?

[Au13]: Update? 